\title{
Estudo Bibliométrico sobre Educação em Engenharia de Materiais
}

DOI: 10.37702/2175-957X.COBENGE.2021.3723

Humberto Dias - humbertodaf@outlook.com

UFSCar

Rua Edmar Neves, 550

39800-350 - Teófilo Otoni - MG

Mariana Salgado Lopes - lopes_ms@outlook.com

Universidade Federal de São Carlos

Rua Abrahão João 435

13562-150 - São Carlos - SP

Daniel Rodrigo Leiva - daniel.leiva@ufscar.br

Universidade Federal de São Carlos

Rodovia Washington Luis, km 235 ---

13565-905 - São Carlos - SP

MARIA ANGELICA DO CARMO ZANOTTO - angelicazanotto@gmail.com Universidade Federal de São Carlos

AV. PARQUE FABER 255

13561-380 - SÃO CARLOS - SP

Leandro Innocentini Lopes de Faria - leandro@ufscar.br

Universidade Federal de São Carlos

Rodovia Washington Luis, km 235 -

13565-905 - São Carlos - SP

Resumo: Vivemos uma crescente oferta nos cursos de engenharia ao redor do mundo, bem como, a necessidade de conhecimentos técnicos correlatos, deficiências e novas demandas vêm sendo enfrentadas na busca por uma transmissão de conhecimento mais eficiente. A Engenharia de Materiais atua promovendo um melhor aproveitamento dos materiais, desenvolvendo novos e agregando noções tecnológicas a setores econômicos estratégicos, aumentando a geração de riquezas, através da criação de empregos e incremento das exportações. Visando apontar direcionamentos e soluções para tal problemática, 
pesquisadores ao redor do mundo têm se dedicado a publicar estudos científicos relacionados ao tema. Nacionalmente, o "Programa Brasil-Estados Unidos de Modernização da Educação Superior na Graduação (PMG-EUA)", possui em seus objetivos o conhecimento de metodologias de ensino e gestão de cursos que aplicáveis à realidade dos cursos superiores brasileiros e a melhoria da educação e adequação às tendências mundiais da Engenharia a partir da criação de redes de colaboração entre o Brasil e os EUA. O presente trabalho insere-se neste contexto com o objetivo de aplicar técnicas bibliométricas em dados recuperados da base de dados elaborando indicadores científicos sobre a Educação em Engenharia de Materiais, identificando as principais instituições, regiões, grupos de pesquisa, fontes de publicação e as tendências referentes ao tema. Constatou-se que os EUA são o país com maior atividade na área, sendo o país líder em número de publicações durante o período analisado. Essa tendência se mantém em relação aos autores com maior número de publicações indexadas a Web of Science para o período analisado.

Palavras-chave: Educação em Engenharia. Bibliometria. Produção Científica. Engenharia de Materiais. 


\section{ESTUDO BIBLIOMÉTRICO SOBRE EDUCAÇÃO EM ENGENHARIA DE MATERIAIS}

\section{INTRODUÇÃO}

Muito vem sendo discutido nos últimos anos sobre a necessidade de readequação do ensino de ensino de engenharia para atender as demandas do setor produtivo, bem como para melhor atender aos próprios estudantes, de forma a resultar em aprendizados mais engajantes e impactantes. Assim sendo, torna-se necessário um maior entendimento sobre o panorama atual do ensino de engenharia no Brasil e no mundo, de forma a determinar instituições e autores relevantes ao tema, detectar pontos para melhoria no ensino, divulgar boas práticas na área e otimizar a destinação de recursos para o fomento das pesquisas científicas sobre o tema (CNI et al., 2020; MARCOS; LEIVA, 2019).

É de conhecimento geral que a educação em engenharia apresenta um papel central no desenvolvimento socioeconômico dos países devido a sua capacidade de formar profissionais responsáveis pela absorção e criação de novas tecnologias. Desta forma, a Engenharia de Materiais, por exemplo, foi introduzida no Brasil com a missão de promover um melhor aproveitamento dos materiais conhecidos, desenvolver novos materiais e agregar noções tecnológicas a setores econômicos estratégicos, aumentando a geração de riquezas do país, através da criação de empregos e aumento das exportações (SAVIGNANO, 2012; UNIVERSIDADE FEDERAL DE SÃO CARLOS, 2004, 2014).

Uma forma bastante empregada na avaliação do impacto da pesquisa científica e na análise de seus resultados é a utilização dos indicadores de ciência e tecnologia. Neste contexto, a bibliometria se apresenta como uma ferramenta bastante útil, por permitir a partir do uso de técnicas estáticas e de inferência e medição, a construção de indicadores quantitativos sobre os registros de publicações científicas de uma área de interesse indexados em bases de dados. Funciona essencialmente como uma mineração de textos capaz de gerar estatísticas a partir de termos recorrentes, sejam estes termos: autores, instituições, ou parte da essência da temática abordada (HOFFMANN et al., 2005; OKUBO, 1997; PINTO; MATIAS, 2011).

Podemos destacar ainda a grande importância das técnicas bibliométricas na identificação das tendências de pesquisa; crescimento do interesse em diferentes disciplinas; estimativa da cobertura das revistas secundárias; aproximação entre autores e usuários das diferentes áreas; identificação dos principais periódicos de cada área; medição da usabilidade dos serviços de disseminação seletiva da informação; construção da cronologia e histórico de tendências de publicação; desenvolvimento de modelos experimentais correlatos ou similares; formulação de políticas de aquisição baseadas na necessidade com provisão de limites orçamentários; adaptação de uma política cuidadosa de armazenagem e descarte; criação de sistemas efetivos de rede de trabalho multinível; regulamentação da entrada de informação e comunicação; previsão da produtividade de editores, autores individuais, organizações e países; esquematização do processamento de linguagem automática para auto-indexação, autoclassificação e autorresumo e; no desenho de normas de padronização (BUFREM, 2011).

Com base no presente exposto, este trabalho propõe a elaboração e análise de indicadores da pesquisa científica sobre o tema "ensino em engenharia dos materiais" presentes na base de dados Web of Science (WoS). Alguns dos dados a serem analisados são: número de publicações por ano, crescimento no número de publicações, países, instituições e autores mais relevantes e tendências de pesquisas na área. 


\section{METODOLOGIA}

A primeira etapa deste estudo baseou-se na recuperação de registros sobre a produção científica em "Educação em Engenharia de Materiais", indexada na WoS. Esta base de dados foi selecionada devido à sua abrangência, multidisciplinaridade, extenso acervo de periódicos revisados por pares, divisão de assuntos em categorias e facilidade na condução de buscas, constituindo a base tradicionalmente mais utilizada. Além disso, possui a maior abrangência e qualidade de recursos disponíveis para tratamento dos dados no Núcleo de Informação Tecnológica em Materiais (NIT/Materiais), localizado no Departamento de Engenharia de Materiais da Universidade Federal de São Carlos.

Em uma das primeiras tentativas, foi aplicado o termo "materials engineering education" no mecanismo de pesquisa básica presente na plataforma. Esta pesquisa retornou cerca de 4.217 resultados, porém por se tratar de um termo genérico, em uma análise mais aprofundada foi verificado que muitos dos artigos presentes nos resultados, por algum motivo não se encaixavam nas pretensões da pesquisa. Podemos tomar como exemplo o trabalho intitulado "Fifty Years of Biomedical Engineering Undergraduate Education", em que a partir do título já é possível perceber que, apesar de dizer respeito à educação em engenharia, trata de tal assunto no viés da Engenharia Biomédica e não da Engenharia de Materiais, como buscado neste trabalho. Em outros casos, os resultados eram ainda mais distantes do desejado, retornando resultados de áreas distintas, como: filosofia, sociologia, humanidade, comunicação e etc.

Dando seguimento ao estudo, iniciaram-se as etapas referentes à construção de uma expressão de busca mais robusta, capaz de recuperar a maioria das publicações relevantes ao tema, dentre a totalidade de registros disponíveis. Vale salientar que este estudo não constitui uma revisão sistemática, sendo assim, não há a ambição de chegar a uma expressão de busca que recupere todos e apenas os artigos relevantes. O intuito deste estudo é obter uma expressão de busca com precisão e revocação suficientes, de forma que permita a construção de indicadores bibliométricos que representem o comportamento geral do campo de pesquisa estudado, tal como em um ensaio mecânico faz-se uso de uma amostra do material, para prever as propriedades de toda a peça.

Como o ensino de Engenharia de Materiais se encontra na fronteira entre os campos de Educação em Engenharia e Engenharia de Materiais, foi utilizada uma estratégia conhecida como "Building Block" para a construção da expressão de busca, em que o pesquisador divide a sua necessidade de informação em diferentes grupos, de acordo com o tema pesquisado. Neste caso, foram utilizados dois grupos diferentes: o bloco de educação e o bloco de materiais. Em cada grupo foi feita uma consulta, gerando diferentes termos e conectados com um operador OR (por exemplo, engenharia de materiais OR seleção de materiais OR ciência dos materiais OR...). Ao final da construção de cada bloco, os diferentes grupos são conectados utilizando o operador AND (por exemplo, seleção de materiais OR ciência dos materiais AND educação OR ensino) (SCHLOSSER et al., 2006).

Já para a seleção dos termos presentes em cada um dos blocos foi empregada a técnica denominada "Pearl Growning", que envolve pesquisas feitas em SmartCat ou bancos de dados, utilizando inicialmente os termos com maior afinidade com o assunto. Após a análise desta pesquisa inicial, novos e melhores termos relevantes ao tema serão encontrados. Desta maneira, novos termos são adicionados aos blocos até que haja um esgotamento dos termos relevantes ao tema pesquisado (SCHLOSSER et al., 2006).

Após o esgotamento das testagens e a inclusão dos termos referentes ao tema, bem como das categorias disponíveis na própria base de dados, a expressão de busca foi estipulada, como pode ser visto no quadro 1. 
Quadro 1- Expressão final de busca.

((TI=("materials science") OR TI=("materials engineer") $) \mathrm{OR} \mathrm{TI=("materials} \mathrm{selection")} \mathrm{OR} \mathrm{TI=("metallurgy")}$ OR TI=("polymer science") OR TI=("ceramic material") OR TI=(metal) OR TI=("alloy*") OR TI=("steel") OR $\mathrm{Tl}=$ ("aluminium") OR TI=("aluminum") OR TI=("polymer*") OR TI=("composite* material"") OR TI=(casting*) OR TI=(foundr*) OR TI=("heat treatment") OR TI=("rheology") OR TI=("tribology")) AND WC=("educat*") ) OR WC=("educat" $\left.{ }^{*}\right)$ AND (WC $=\left(" m a t e r i a l^{*}\right.$ science") OR WC=(" polymer science") OR WC=("metallugy \& metallurgical engineering")) OR TI= (("educat" NEAR/2 "materials scince") OR ("educat" ${ }^{*}$ NEAR/2 "materials engineer*") OR ("educat*" NEAR/2 "materials selection") OR ("educat*" NEAR/2 "metallurg*") OR ("educat" NEAR/2 "polymer science") OR ("educat*" NEAR/2 "ceramic material*") OR ("educat*" NEAR/2 "metal") OR ("educat" NEAR/2 alloy*) OR ("educat" NEAR/2 "steel") OR ("educat" NEAR/2 "aluminium") OR ("educat*" NEAR/2 "aluminum") OR ("educat*" NEAR/2 "polymer*") OR ("educat*" NEAR/2 "composite* material*") OR ("educat" NEAR/2"foundr") OR ("educat" NEAR/2 "heat treatment") OR ("educat*" NEAR/2 "rheology") OR ("educat*" NEAR/2 "tribology")) OR TI= (("teach" NEAR/2 "materials scince") OR ("teach*" NEAR/2 "materials engineer*") OR ("teach*" NEAR/2 "materials selection") OR ("teach*" NEAR/2 "metallurg*") OR ("teach*" NEAR/2 "polymer science") OR ("teach*" NEAR/2 "ceramic material*") OR ("teach*" NEAR/2 "metal") OR ("teach" NEAR/2 alloy*) OR ("teach*" NEAR/2 "steel") OR ("teach*" NEAR/2 "aluminium") OR ("teach*" NEAR/2 "aluminum") OR ("teach*" NEAR/2 "polymer*") OR ("teach*" NEAR/2 "composite* material*") OR ("teach" NEAR/2 "foundr*") OR ("teach" NEAR/2 "heat treatment") OR ("teach*" NEAR/2 "rheology") OR ("teach*" NEAR/2 "tribology")) OR TI=((("learning" NEAR/2 "materials scince") OR ("learning" NEAR/2 "materials engineer*") OR ("learning" NEAR/2 "materials selection") OR ("learning" NEAR/2 "metallurg*") OR ("learning" NEAR/2 "polymer science") OR ("learning" NEAR/2 "ceramic material" ) OR ("learning" NEAR/2 "metal") OR ("learning" NEAR/2 alloy*) OR ("learning" NEAR/2 "steel") OR ("learning" NEAR/2 "aluminium") OR ("learning" NEAR/2 "aluminum") OR ("learning" NEAR/2 "polymer*") OR ("learning" NEAR/2 "composite* material*") OR ("learning" NEAR/2 "foundr" ) OR ("learning" NEAR/2 "heat treatment") OR ("learning" NEAR/2 "rheology") OR ("learning" NEAR/2 "tribology")) NOT ("machine learning" OR "deep learning")) OR TI= (("classroom" NEAR/2 "materials science") OR ("classroom" NEAR/2 "materials engineer*") OR ("classroom" NEAR/2 "materials selection") OR ("classroom" NEAR/2 "metallurg*") OR ("classroom" NEAR/2 "polymer science") OR ("classroom" NEAR/2 "ceramic material*") OR ("classroom" NEAR/2 "metal") OR ("classroom" NEAR/2 alloy*) OR ("classroom" NEAR/2 "steel") OR ("classroom" NEAR/2 "aluminium") OR ("classroom" NEAR/2 "aluminum") OR ("classroom" NEAR/2 "polymer*") OR ("classroom" NEAR/2 "composite* material*") OR ("classroom" NEAR/2 "foundr*") OR ("classroom" NEAR/2 "heat treatment") OR ("classroom" NEAR/2 "rheology") OR ("classroom" NEAR/2 "tribology") ) OR $\mathrm{Tl}=$ (("curricul*" NEAR/2 "materials science") OR ("curricul" NEAR/2 "materials engineer*") OR ("curricul" NEAR/2 "materials selection") OR ("curricul" NEAR/2 "metallurg"") OR ("curricul*" NEAR/2 "polymer science") OR ("curricul*" NEAR/2 "ceramic material*") OR ("curricul*" NEAR/2 "metal") OR ("curricul*" NEAR/2 alloy*) OR ("curricul*" NEAR/2 "steel") OR ("curricul*" NEAR/2 "aluminium") OR ("curricul" NEAR/2 "aluminum") OR ("curricul" NEAR/2 "polymer*") OR ("curricul" NEAR/2 "composite* material*") OR ("curricul" NEAR/2 "foundr*") OR ("curricul*" NEAR/2 "heat treatment") OR ("curricul*" NEAR/2 "rheology") OR ("curricul" NEAR/2 "tribology") ) OR TI= (("student*" NEAR/2 "materials science") OR ("student" NEAR/2 "materials engineer") OR ("student" NEAR/2 "materials selection") OR ("student*" NEAR/2 "metallurg*") OR ("student*" NEAR/2 "polymer science") OR ("student ${ }^{* "}$ NEAR/2 "ceramic material" ) OR ("student*" NEAR/2 "metal") OR ("student*" NEAR/2 alloy*) OR ("student*" NEAR/2 "steel") OR ("student*" NEAR/2 "aluminium") OR ("student*" NEAR/2 "aluminum") OR ("student" NEAR/2 "polymer*") OR ("student" NEAR/2 "composite* material") OR ("student" NEAR/2 "foundr") OR ("student*" NEAR/2 "heat treatment") OR ("student*" NEAR/2 "rheology") OR ("student" NEAR/2 "tribology") ) OR Tl= (("training" NEAR/2 "materials science") OR ("training" NEAR/2 "materials engineer*") OR ("training" NEAR/2 "materials selection") OR ("training" NEAR/2 "metallurg*") OR ("training" NEAR/2 "polymer science"))

Fonte: Autoria própria, 2021.

Por fim, foi realizado um último refinamento, selecionando apenas os artigos indexados no período de 2001 a 2020, permitindo a construção de um panorama que representa as tendências atuais deste campo de pesquisa e que ainda possui uma quantidade suficiente de registros para entender a dinâmica desta área ao longo do tempo. O tamanho e robustez da expressão de busca, demonstrou o cuidado e complexidade envolvidos em seu processo de construção, garantindo o equilíbrio entre abrangência e 
especificidade mais adequado à visão dos autores. Nestas condições, a pesquisa retornou um total de 2.349 registros (MARCOS, 2020).

Após todo o processo descrito, os dados obtidos foram exportados em arquivos de texto e tratados utilizando o software VantagePoint v5.0. O tratamento incluiu a padronização dos resultados obtidos como: nomes dos autores, instituições de ensino e países das publicações. Posteriormente, foi utilizado o software de planilhas eletrônicas Microsoft Excel para a construção dos gráficos apresentados na seção a seguir.

\section{RESULTADOS E DISCUSSÃO}

Dando início à análise dos resultados, os dados a respeito do número de publicações anuais indexadas à base de dados WoS, no período de 2001 a 2020, estão apresentados na figura 1.

Figura 1 - Número de publicações por ano nos últimos 20 anos (2001-2020).

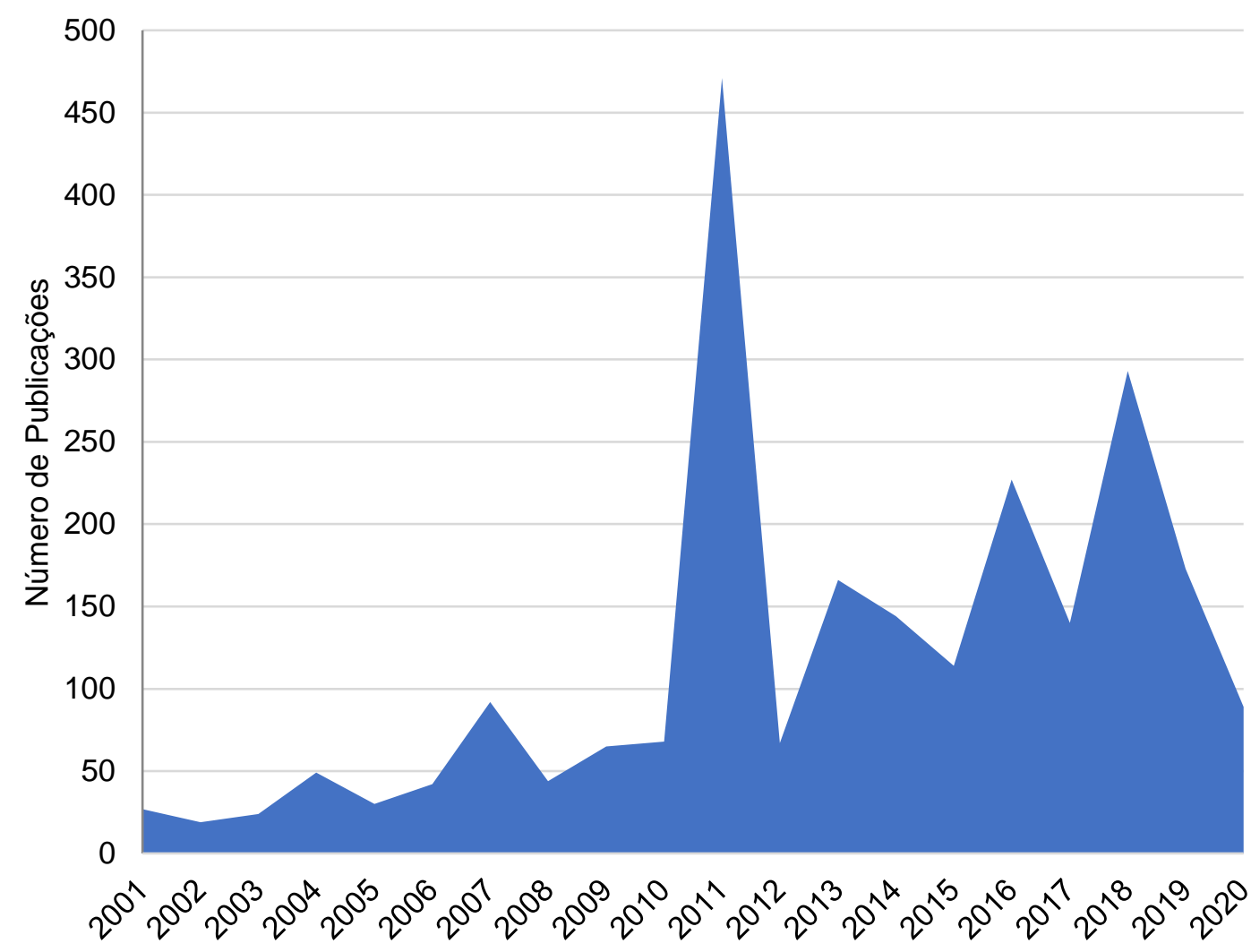

Fonte: Os autores, 2021.

Nos primeiros anos analisados, podemos observar um número relativamente baixo de publicações para o tema, com valores de até 100 publicações por ano. Após esta fase, pode-se observar uma tendência de crescimento nos anos de 2011, 2013, 2016 e 2018. É notável a quantidade de publicações indexadas à base de dados no ano de 2011, com destaque para a grande quantidade de trabalhos publicados pela ADVANCED MATERIALS RESERCH, compondo 388 publicações das 471 observadas no período. A segunda fonte de publicações para o ano analisado, JOURNAL OF MATERIALS EDUCATION, teve apenas 20 publicações indexadas à base de dados. Ambos os periódicos apresentam grande relevância ao tema Engenharia de Materiais, justificando sua presença entre os resultados encontrados e o crescimento observado. 
Por sua vez, em 2018, ano com o segundo maior número de publicações, houve uma grande indexação de trabalhos de importantes congressos, como o IOP CONFERENCE SERIES MATERIAL SCIENCE AND ENGINEERING, destacando a importância da realização de eventos científicos para a divulgação científica, troca de informações entre pesquisadores e geração de registros sobre direcionamentos de pesquisas.

Dando prosseguimento, a figura 2 ilustra em ordem de número de publicações, os países mais ativos em termos de publicações a respeito do tema abordado. O primeiro ponto a ser considerado está na clara predominância dos Estudos Unidos como país líder em publicações a respeito de Educação em Engenharia. O país detém 527 publicações para o intervalo de tempo analisado, enquanto a China, que ocupa a segunda posição detêm 377 publicações e a Indonésia, em $3^{\circ}$ lugar possui 196 publicações. O Brasil, embora ocupe a 10ª posição, possui apenas 34 publicações no tema para o período analisado.

Figura 2 - Número de publicações por país, para o período de 2001 a 2020.

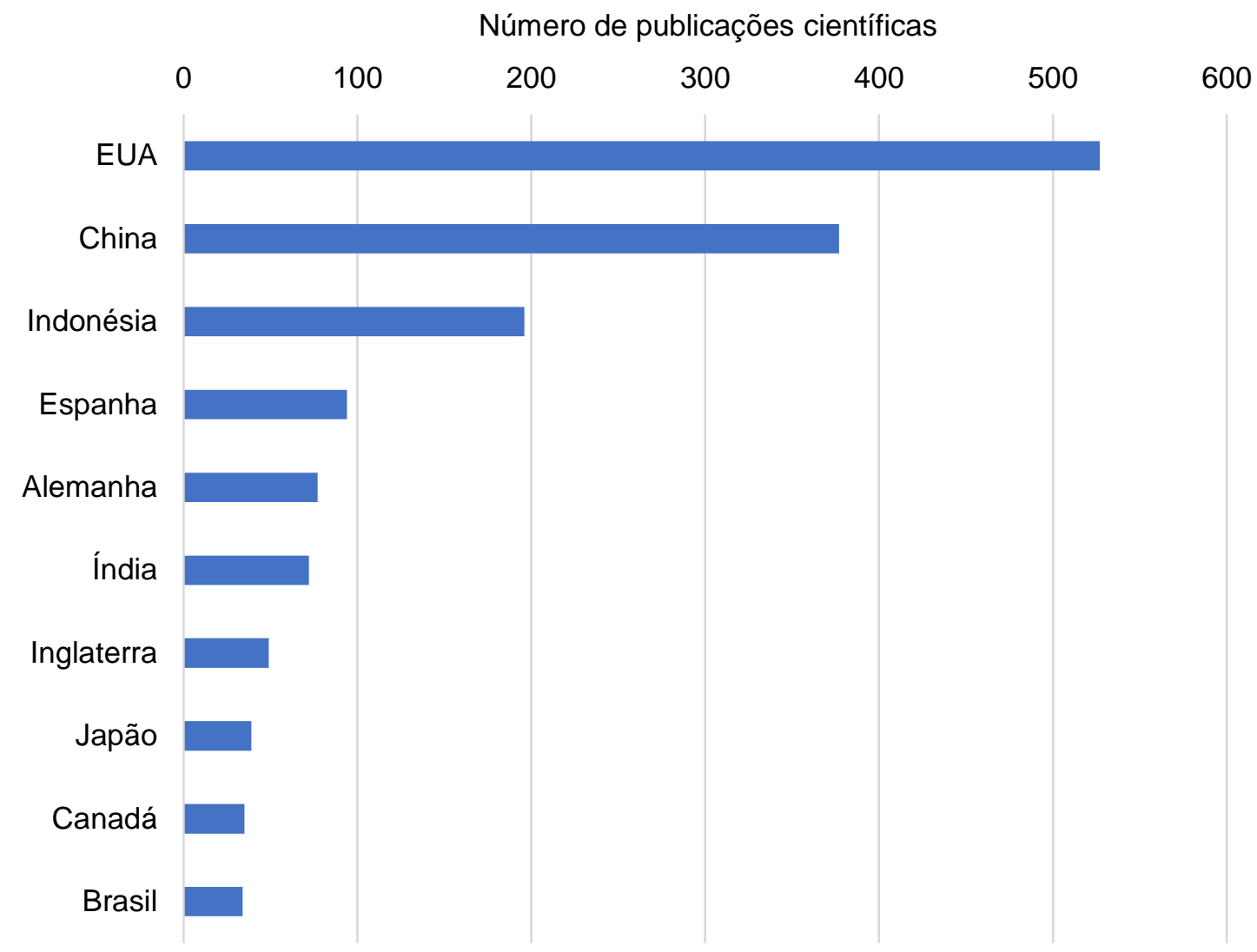

Fonte: Os autores, 2021.

Cabe mencionar ainda que uma publicação é atribuída a um país, quando ao menos um de seus autores apresenta a nacionalidade referida, podendo, portanto, uma mesma publicação ser atribuída a vários países diferentes, de acordo com a nacionalidade de seus autores e instituições. Desta maneira, torna-se importante entender quais instituições apresentam maior notoriedade no tema buscado, como mostra a figura 3. 
Figura 3 - Número de publicações por instituição de ensino, para o período de 2001 a 2020.

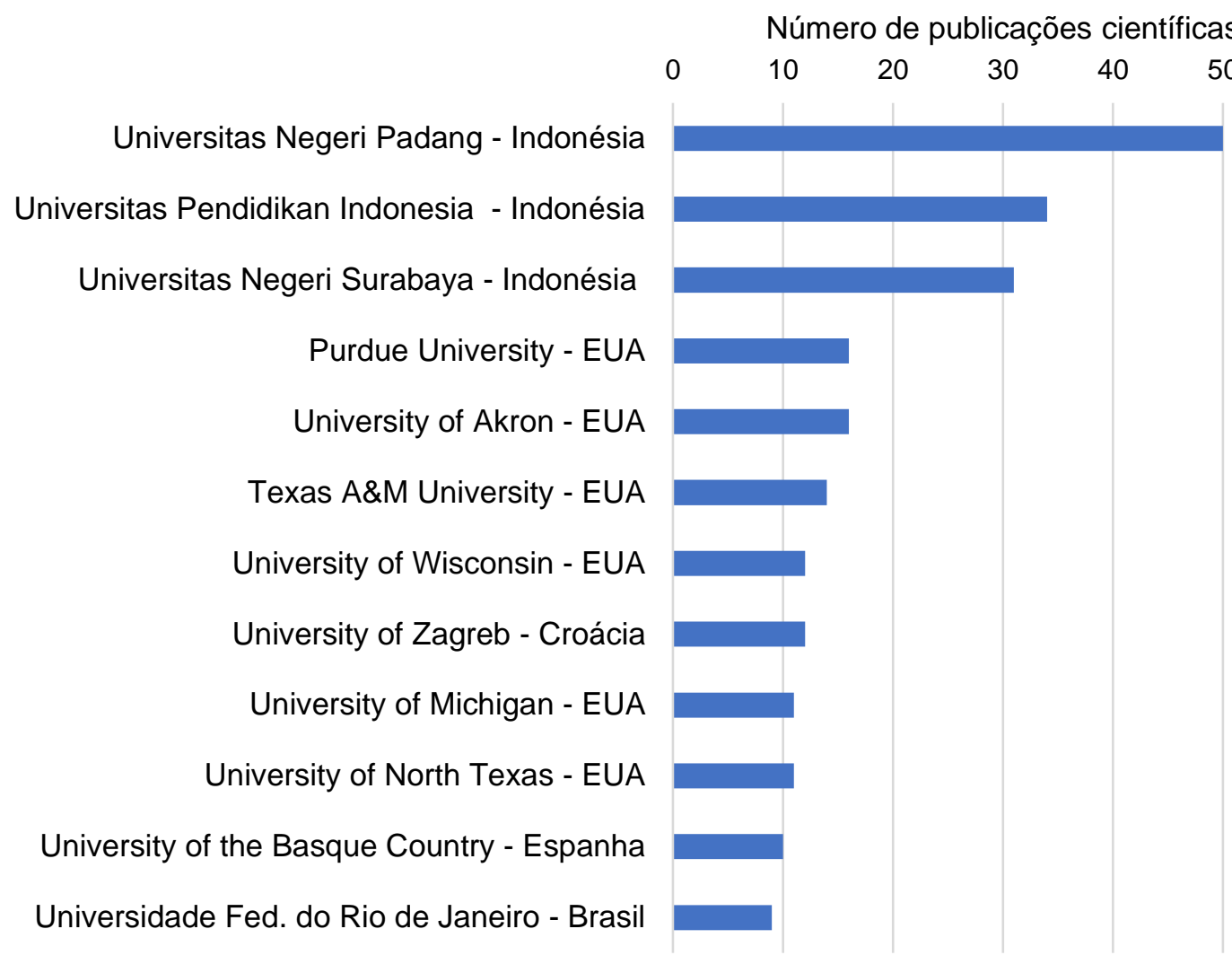

Fonte: Os autores, 2021.

É interessante observar que embora os três maiores números de publicações pertençam a instituições localizada na Indonésia, a dominância dos EUA na área de educação em engenharia de materiais se mantém, uma vez que entre as dez instituições com maior número de publicações, seis delas são estadunidenses, enquanto apenas três instituições são indonésias e uma é croata.

A instituição brasileira com melhor posicionamento foi a Universidade Federal do Rio de Janeiro, ocupando a $12^{a}$ posição, com nove trabalhos indexados na área de materiais poliméricos, sendo oito deles publicados na revista JOURNAL OF MATERIALS EDUCATION e um na MOLECULAR SYMPOSIA.

Foi realizado ainda um agrupamento do conjunto das palavras-chave com maior ocorrência nas publicações recuperadas pela busca, apresentado na figura 4. Este levantamento teve como objetivo um maior entendimento a respeito dos assuntos mais estudados pela área de educação em engenharia de materiais. 
Figura 4 - Palavras-chave de maior ocorrência.

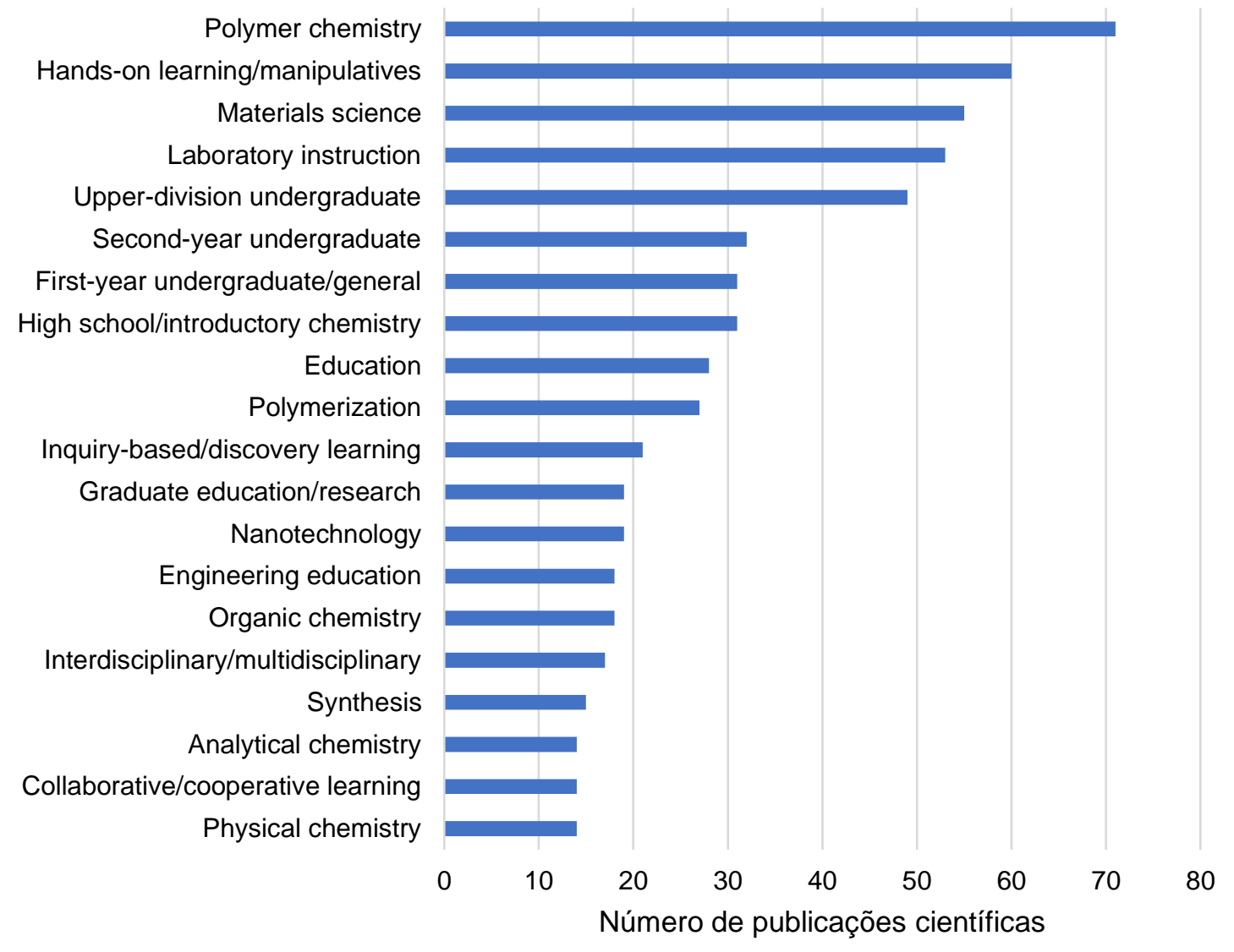

Fonte: Os autores, 2021.

Pode-se perceber a presença de alguns termos bem genéricos, como "Education", "Nanotchnology", "Synthesis", entre outros, que separadamente não nos permitem aprofundar tanto em sua análise. Por outro lado, o ensino na área de materiais poliméricos tem se mostrado bastante ativo nas publicações a respeito do ensino em Engenharia de Materiais, tendo em vista a recorrente presença das seguintes palavras-chave: "Polymer chemistry", "Polymerization", e "Organic Chemistry".

É possível observar também a ocorrência de palavras-chave relacionadas às metodologias ativas de ensino-aprendizagem, importantes para a modernização do ensino, uma vez que estas substituem os tradicionais métodos de transmissão de conteúdo do professor para o aluno, dando ao estudante o protagonismo de seu processo formativo, permitindo um maior engajamento e direcionamento da formação de acordo com os interesses profissionais almejados.

O primeiro termo que aparece e que está relacionado às metodologias ativas de aprendizagem é "Hands-on learning/manipulatives", quando os alunos fazem parte do processo e estão envolvidos na aprendizagem ativa, praticando suas habilidades e colocando seus conhecimentos à prova. O segundo termo é o "Inquiry based/discovery learning", que é um método de instrução baseado em pesquisa. Esta teoria incentiva os alunos a construírem sobre experiências e conhecimentos passados, usar sua intuição, imaginação e criatividade, além de procurar por novas informações para descobrir fatos, correlações e novas verdades $O$ terceiro termo que aparece é "Collaborative/cooperative learning", que constitui um recurso na área de educação que advém da necessidade de 
inserir metodologias interativas entre os estudantes, em conjunto com o professor para que estabeleçam buscas, compreensão e interpretação da informação de assuntos determinados.

A figura 5 apresenta um panorama geral sobre autores com maior número de publicações na área.

Figura 5 - Número de publicações no período de 2001 a 2020.

Número de publicações científicas

$\begin{array}{llllllll}0 & 2 & 4 & 6 & 8 & 10 & 12 & 14\end{array}$

Choi, S. R.

Wang, Y. Z.

Morscher, G. N.

Wang, J. Y.

Wang, X. Z.

Li, Y. Y.

Liu, J. X.

Abdi, F.

Brostow, W.

Castano, V. M.

Fonte: Os autores, 2021.

Dos dez nomes presentes na lista de principais autores, quatro são estadunidenses ou atuam no país. Com o mesmo número de autores figurando entre os principais, a China também mostra sua relevância em relação ao tema. Essa predominância dos EUA e China, faz todo sentido se levarmos em conta, como vimos na Figura 2, que estes são os dois principais países em número de publicações para o período estudado. Já os demais autores que estão listados acima são naturais ou atuam no Canadá e México.

O principal nome que aparece entre os listados é o do autor Choi, S. R., autor estadunidense que possui 13 publicações nas áreas de materiais compósitos e caracterização de materiais. As publicações do autor estão ligadas à instituição Naval Air Systems Command.

Outro importante autor dos EUA que aparece na terceira posição em número total de publicações é Morscher, G. N., membro da comunidade acadêmica da University of Akron, que está entre as principais instituições de ensino observadas anteriormente na figura 3. Outros dois autores estadunidenses que estão relacionados são: Abdi F. e Brostow, W., possuindo 8 publicações cada.

Já os autores Wang, Y. Z.; Wang, J. Y. e Wang, X. Z., figuram na segunda (12 publicações), quarta (11 publicações) e quinta (11 publicações) posições respectivamente. Os autores são membros da comunidade acadêmica Chengdu Technol University, localizada na China. Outro autor chinês na listagem é Li, Y. Y., que figura na sexta posição com 10 publicações. 


\section{CONCLUSÃO}

O estudo bibliométrico realizado teve como finalidade obter uma visão geral acerca da situação e direcionamentos sobre as pesquisas em educação em Engenharia de Materiais a partir dos registros indexados à base de dados Web of Science, tornando possível uma melhor contextualização da produção científica na área, em uma esfera mundial e identificação de possíveis tendências, além de revelar os principais autores e instituições atuantes na área.

Mostrou-se notória a dominância dos EUA nessa temática de estudo, sendo o país líder em número de publicações, durante o período analisado. Essa tendência se mantém em relação aos autores com maior número de publicações. Além disso, entre os dez autores com maior número de publicações, metade atua nos EUA. A China também apresenta bastante participação em pesquisas na temática, figurando como segundo pais em maior número de publicações para o período analisado, bem como, possui três entre os quatro maiores pesquisadores na área.

Observou-se também que o Brasil é responsável por apenas uma pequena parcela das publicações retornadas, não constituindo, deste modo, um país de destaque na área de educação em Engenharia de Materiais até o momento.

Quanto à análise das palavras-chave que mais se repetem, notou-se uma grande ocorrência de termos ligados às metodologias ativas de aprendizagem.

Como propostas para trabalhos futuros temos a realização de uma busca similar em outras bases de dados, mais específicas para a área de engenharia, como a Engineering Village ou Compendex, que poderia complementar as informações, dado que possui uma maior abrangência dentro do contexto de engenharia. Um maior aprofundamento dos dados poderia ser feito com intuito de investigar as redes de colaboração entre autores e instituições e analisar correlações entre palavras-chave e instituições para determinar os assuntos específicos trabalhados.

\section{Agradecimentos}

Ao CNPq - Conselho Nacional de Desenvolvimento Científico e Tecnológico pelo apoio financeiro para realização desse trabalho com bolsa de estudos, processo 143364/2019-7.

À CAPES - Coordenação de Aperfeiçoamento de Pessoal de Nível Superior pelo apoio financeiro para realização desse trabalho com bolsa de estudos, processo no 88887.494624/2020-00.

O presente trabalho foi realizado com apoio da Coordenação de Aperfeiçoamento de Pessoal de Nível Superior - Brasil (CAPES) - Código de Financiamento 001.

\section{REFERÊNCIAS}

BRASIL, Ministério da Educação PROCESSO №: 23001.000141/2015-11. Diretrizes Curriculares Nacionais do Curso de Graduação em Engenharia. Diário Oficial da União: seção 1, Brasília, DF, p. 109, 23 abr. 2019.

BUFREM, Leilah Santiago; GABRIEL JUNIOR, Rene Faustino; SORRIBAS, Tidra Viana. Redes sociais na pesquisa científica da área de ciência da informação. DataGramaZeroRevista de Informação, v. 12, n. 3, 2011. 
CAPES - Coordenação de Aperfeiçoamento de Pessoal de Nível Superior. EDITAL no 23/2018: "Programa Brasil-Estados Unidos de modernização da educação superior na graduação (PMG-EUA)". Brasília: CAPES/CNE/COMISSÃO FULBRIGHT, 2018.

Disponível em: https://www.capes.gov.br/images/stories/download/editais/11062018Edital_23_PMG_EUA2.pdf. Acesso em: 27 apr. 2021.

CNI; SESI; SENAI; IEL; CNE; ABENGE; CONFEA. Documento de apoio à implantação das DCNs do curso de graduação em engenharia. Brasília: CNI, 2020. Disponível em: https://static.portaldaindustria.com.br/media/filer_public/95/2b/952b2b44-3671-4556-bfa8d0f446185a29/documento_de_apoio_implantacao_dcns_curso_graduacao_engenharia.pd f.

HOFFMANN, W. A. M.; FARIA, L. I. L. De; QUONIAM, L.; QUEYRAS, J. Análise da produção científica a partir de indicadores bibliométricos. In: GREGOLIN, J. Â. R. (Ed.). Indicadores de ciência, tecnologia e inovação em São Paulo 2004. São Paulo - SP: FAPESP, 2005. v. 1p. 5-43.

MARCOS, L. P. PERSPECTIVAS E ESTRATÉGIAS PARA MELHORIA DA EDUCAÇÃO EM ENGENHARIA DE MATERIAIS BRASILEIRA. 2020. Dissertação - Universidade Federal de São Carlos, São Carlos, 2020.

MARCOS, L. P.; LEIVA, D. R. Indicadores Bibliométricos de Publicações sobre Educação em Engenharia. In: FORMAÇÃO POR COMPETÊNCIA NA ENGENHARIA NO CONTEXTO DA GLOBALIZAÇÃO 4.0 2019, Fortaleza, CE. Anais. In: CONGRESSO BRASILEIRO DE EDUCAÇÃO EM ENGENHARIA. Fortaleza, CE Disponível em: http://www.abenge.org.br/sis_submetidos.php?acao=abrir\&evento=COBENGE19\&codigo =COBENGE19_00126_00002313.pdf.

OKUBO, Y. Bibliometric indicators and Analysis of research systems: OECD Science, Technology and Industry Working Papers. Paris. [s.n.]. Disponível em: https://www.oecdilibrary.org/science-and-technology/bibliometric-indicators-and-analysis-of-researchsystems_208277770603. Acesso em: 16 jun. 2020.

PINTO, A. L.; MATIAS, M. Indicadores Científicos e as Universidades Brasileiras. Informação \& Informação, [s. I.], v. 16, n. 3, p. 1-18, 2011. Disponível em: http://www.uel.br/revistas/uel/index.php/informacao/article/view/11498. Acesso em: 11 jul. 2020.

SAVIGNANO, V. Um aniversário da Engenharia de Materiais no Brasil. Sociedade Brasileira de Pesquisa em Materiais. Rio de Janeiro, 2012. Disponível em: https://www.sbpmat.org.br/pt/um-aniversario-da-engenharia-de-materiais-no-brasil-40anos-do-dema-da-ufscar/. Acesso em: 15 fev. 2021.

SCHLOSSER, R. W.; WENDT, O.; BHAVNANI, S.; NAIL-CHIWETALU, B. Use of information-seeking strategies for developing systematic reviews and engaging in evidence-based practice: the application of traditional and comprehensive Pearl Growing. A review. International Journal of Language \& Communication Disorders, [s. I.], v. 41, n. 5, p. 567-582, 2006. Disponível em: http://doi.wiley.com/10.1080/13682820600742190. Acesso em: 9 maio. 2021. 
UNIVERSIDADE FEDERAL DE SÃO CARLOS. Projeto Pedagógico de Curso, 2004.

UNIVERSIDADE FEDERAL DE SÃO CARLOS. Primeiro curso da UFSCar e da América Latina, a Engenharia de Materiais se destaca na formação de profissionais atuantes na academia e no mercado de trabalho. Institucional. 2014. Disponível em: http://www.dema.ufscar.br/portal/index.php/advanced-stuff/o-curso. Acesso em: 25 jan. 2021.

\section{BIBLIOMETRIC STUDY OF MATERIALS ENGINEERING EDUCATION}

Abstract: We are experiencing a growing supply of engineering courses around the world, as well as the need for related technical knowledge, deficiencies and new demands are being faced in the search for a more efficient knowledge transmission. Materials Engineering works promoting a better use of materials, developing new ones and adding technological notions to strategic economic sectors, increasing the generation of wealth, through the creation of jobs and increase in exports. Aiming to point out directions and solutions to this problem, researchers around the world have been dedicated to publishing scientific studies related to the theme. Nationally, the "Brazil-United States Program for the Modernization of Higher Education in Graduation (PMG-EUA)", has in its objectives the knowledge of teaching methodologies and course management that are applicable to the reality of Brazilian higher education courses and the improvement of education and adaptation to the world trends in Engineering based on the creation of collaboration networks between Brazil and the USA. The present work is inserted in this context with the objective of applying bibliometric techniques in data recovered from the database, elaborating scientific indicators about Education in Materials Engineering, identifying the main institutions, regions, research groups, sources of publication and trends related to the theme. It was found that the USA is the country with the most activity in the area, being the leading country in number of publications during the analyzed period. This trend is maintained in relation to the authors with the highest number of publications indexed to Web of Science for the analyzed period.

Keywords: Engineering Education. Bibliometrics. Scientific Production. Materials Engineering. 